\title{
A FIXED POINT THEOREM FOR NILPOTENT LIE GROUPS
}

\section{LOUIS AUSLANDER}

At present, little general information seems to be available concerning the fixed points of compact abelian groups operating on Euclidean $n$ dimensional space, $E^{n}$. In view of this fact, it is perhaps worthwhile to enlarge our information concerning special cases of such phenomena. One such special case, is the classical theorem that a compact abelian group of affine transformations has a fixed point. It is our intention to generalize this theorem to connected simply connected, nilpotent Lie groups. In order to see clearly the nature of the generalization, we shall first present a description of the affine groups, $A(n)$ and its action on $E^{n}$.

Let $E^{n}$ be considered as a vector space or abelian Lie group. Then $G L(n, R)$ is the group of continuous automorphisms of $E^{n}$ and $A(n)$ is the semi-direct product of $E^{n}$ and $G L(n, R)$. We will denote this by $A(n)=E^{n} \cdot G L(n, R)$ and an element of $A(n)$ will be denoted by $(r, g)$ where $r \in E^{n}$ and $g \in G L(n, R)$. Then multiplication in $A(n)$ is defined by $\left(r_{1}, g_{1}\right)\left(r_{2}, g_{2}\right)=\left(r_{1} g_{1}\left(r_{2}\right), g_{1} g_{2}\right)$. We can also define a mapping $\eta: A(n) \rightarrow E^{n}$ by $\eta(r, g)=r$. Then the action of $A(n)$ on $E^{n}$ is given by $r \rightarrow \eta\left(a_{0} r\right)$ for all $r \in E^{n}$ and $a_{0} \in A(n)$. Now let $N^{n}$ be a connected, simply connected $n$ dimensional nilpotent Lie group. Then $N^{n}$ is homeomorphic to $E^{n}$. Let $G\left(N^{n}\right)$ be the group of continuous automorphisms of $N^{n}$ and define the group $A\left(N^{n}\right)$ as the semi-direct product of $N^{n}$ and $G\left(N^{n}\right)$. Then $A\left(N^{n}\right)=N^{n} \cdot G\left(N^{n}\right)$ and the elements of $A\left(N^{n}\right)$ will be denoted by $(n, g)$ for $n \in N^{n}$ and $g \in G\left(N^{n}\right)$. Then multiplication in $A\left(N^{n}\right)$ is given by $\left(n_{1}, g_{1}\right)\left(n_{2}, g_{2}\right)=\left(n_{1} g_{1}\left(n_{2}\right), g_{1} g_{2}\right)$. We can clearly define $A\left(N^{n}\right)$ operating on $N^{n}$ exactly as in the abelian case of $E^{n}$.

Theorem. Let $B$ be a compact abelian subgroup of $A\left(N^{n}\right)$. Then there is a point $n_{0} \in N$ such that for all $b \in B, b\left(n_{0}\right)=n_{0}$.

Proof. We will prove the theorem by induction on the dimension of $N^{n}$. Clearly the theorem is true for $n=1$. Assume the theorem is true for $n=k$. Let $N^{k+1}$ be given and $B \subset A\left(N^{k+1}\right)$ be a compact abelian group. Then $B$ can be represented as the direct sum of a toral group $T$ and a finite group $F$. If $F$ is the trivial group, then the theorem is true by a result of $P$. A. Smith [1]. Hence we may assume that $F$ is nontrivial with generators $f_{1}, \cdots, f_{r}$. Let $f_{1}=\left(n_{1}, a_{1}\right)$. Then $a_{1}$ cannot be the identity element of $G\left(N^{k+1}\right)$, because $N^{k+1}$ has no ele-

Received by the editors March 16, 1958. 
ments of finite order. Let $M$ be the maximal subgroup of $N^{k+1}$ such that $a_{1} m=m$ for $m \in M$. Then $M$ is a connected, simply connected, nilpotent Lie group. To see that this is the case, we merely have to notice that if $a_{1} m=m$ and $L(m)$ denotes the one parameter subgroup of $N^{k+1}$ through $m$ then $a_{1} L(m)=L(m)$ point-wise. Since $a_{1}$ is not the identity element of $G\left(N^{k+1}\right)$, we have $\operatorname{dim} M<k+1$.

Since $B$ is compact, $a_{1}$ is a semi-simple automorphism of $N^{k+1}$. (For a definition see [2].) Hence by [2], we can find a $y \in N^{k+1}$ such that $a_{1}(y) n_{1} y^{-1} \in M$. If we let $z=a_{1}^{-1}\left(y^{-1}\right)$ we have that $z^{-1} n_{1} a_{1}(z) \in M$. Now consider the inner automorphism of $A\left(N^{k+1}\right)$ induced by $\left(z^{-1}, e\right)$. This defines a mapping $\phi$ of $N^{k+1}$ onto itself and mapping $\phi^{*}$ of $B$ onto a group $B^{*}$ which satisfies the condition $\phi b=b^{*} \phi$, where $b^{*}=\phi^{*} b$. Let $f_{1}^{*}=\phi^{*} f_{1}$. Then $f_{1}^{*}=\left(n_{1}^{*}, a_{1}\right)$ where $n_{1}^{*} \in M$. Hence, since $f_{1}^{*}$ is of finite order in $B, n_{1}^{*}$ must be the identity element of $M$ or $f_{1}^{*}=\left(e, a_{1}\right)$. Now let $b^{*} \in B^{*}$ with $b^{*}=(n, a)$. Since $f_{1}^{*} b^{*}=b^{*} f_{1}^{*}$ we must have that $a_{1} n=n$. Further since $a_{1}$ and $a$ commute, we have that $M$ is mapped onto itself by $a$. Hence $b^{*} \in B^{*}$ maps $M$ onto itself. But $\operatorname{dim} M<k+1$. Hence by induction there exists a point in $M$ which is fixed for all $b^{*} \in B^{*}$. Since $M$ is a subset of $N^{k+1}$, this proves the theorem.

\section{REFERENCES}

1. P. A. Smith, Stationary points of transformation groups, Proc. Nat. Acad. Sci. U.S.A. vol. 28 (1942) pp. 293-297.

2. H. C. Wang, Discrete subgroups of solvable Lie groups, Ann. of Math. vol. 64 (1956) pp. 1-19.

INDIANA UNIVERSITY 